\title{
MULTIPLE FORMS OF LACTIC DEHYDROGENASE IN TISSUES OF THE MOUSE: THEIR SPECIFICITY, CELLULAR LOCALIZATION, AND RESPONSE TO ALTERED PHYSIOLOGICAL CONDITIONS*
}

\author{
John M. Allen \\ Department of Zoology, The University of Michigan, Ann Arbor, Mich.
}

There is increasing evidence that many enzymes exist in tissues and cells in multiple molecular forms (Markert and Møller, 1959). Of less certainty, however, is the question of differences between these multiple molecular forms in terms of their catalytic and physiological activities. Indeed Markert and Møller felt that these forms, termed isozymes, possessed identical substrate specificities although the pattern of the isozymes possessed by a tissue changed radically during development. The question of the degree of heterogeneity existing between the multiple molecular forms of an enzyme is one of considerable importance. Such heterogeneity may be reflected in several diverse ways: (1) differences in substrate specificity, (2) differences in capacity to utilize cofactor analogs, (3) differences in sensitivity to inhibitors, (4) differences in response to varied physiological conditions, or (5) differences in the site of localization within or between cells. An examination of these possible points of difference between the multiple forms of lactic dehydrogenase in the tissues of the mouse comprises the content of this paper.

\section{Laterials and Methods}

All work was carried out using tissues from BALB/c Jax mice. Tissues for electrophoretic study were homogenized in 2 parts $(\mathrm{w} / \mathrm{v}) 0.9$ per cent sodium chloride, using all-glass homogenizers. These homogenates were centrifuged at $4^{\circ} \mathrm{C}$. for $10 \mathrm{~min}$. at $1000 \mathrm{~g}$ to remove cell debris. The supernatant material from this centrifugation was employed for electrophoresis. Electrophoresis was carried out in a starch matrix after the method of Smithies (1959), as modified by Markert and Hunter (1959). The starch matrix $\dagger$ was prepared using $0.03 M$ boric acid-sodium hydroxide buffer at $p \mathrm{H}$ 8.5. The bridge buffer was similar in composition but was 10 times as concentrated. Electrophoresis was carried out for 6 hours at room temperature (ca. $25^{\circ} \mathrm{C}$.), using a voltage drop of $6 \mathrm{v} / \mathrm{cm}$. along the length of the starch slabs. For cytochemical study, $30 \lambda$ of the above supernatant material was applied to a $24 \times 6 \mathrm{~mm}$. strip of Whatman No. 1 filter paper, and the strip was inserted into a transverse slit in the starch slab. For quantitative study of eluted enzyme, $60 \lambda$ of the supernatant was applied to a double-paper insert of the same size.

Cytochemical visualization of sites of lactic dehydrogenase activity in starch slabs was achieved by a modification of the method proposed by Dewey and Conklin (1961). The substrate mixture contained the following final concen-

* 'The work described in this paper was supported in part by Grants C-2751 C-4 from the National Cancer Institute, Public Health Service, Bethesda, Md., and in part by an Institutional Grant to The University of Michigan, Ann Arbor, Mich., from the American Cancer Society, New York, N.Y.

† Obtained from Connaught Medical Research Laboratories, Toronto, Ont., Canada. 
trations of reactants: $0.025 M$ tris(hydroxymethyl)aminomethane* buffer

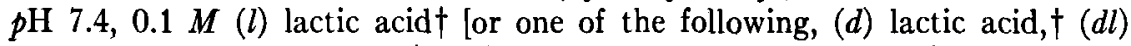
alpha-hydroxybutyric acid,* (dl) alpha-hydroxycaproic acid,* $(d l)$ alphahydroxyisobuteric acid,* $(d l)$ alpha-hydroxyisovaleric acid,* or $(d l)$ betaphenyllactic acid*; $0.001 M$ diphosphopyridine nucleotide (DPN), ${ }^{*}$ or deamino-diphosphopyridine nucleotide (DeamDPN); $0.5 \mathrm{mg}$. per ml. nitro-blue tetrazolium (NBT) $\$ 0.005 M$ potassium cyanide; and $20 \mu$ gm. per ml. phenazine methosulfate (PMS). ${ }^{*}$ Starch slabs for incubation in this substrate mixture were sliced into 2 equal thicknesses with the bottom half always used for analysis. This bottom half was again divided longitudinally into equal halves so that 2 identical strips were available for comparative purposes. These strips were incubated at room temperature $\left(\mathrm{ca} .25^{\circ} \mathrm{C}\right.$.) in total darkness for $30 \mathrm{~min}$. The results were recorded photographically, using constant conditions of exposure and development.

In cases where quantitative estimation of enzyme activity was to be made, a 1-mm. strip was sliced from the edge of the starch slab and incubated in the above substrate mixture [containing $(l)$ lactic acid] for $30 \mathrm{~min}$. or less. This developed strip then served as a guide to the position of the corresponding activity sites in the remaining portion of the starch slab. These sites were cut out, using a jig that produced a $3-\mathrm{mm}$. slice to yield a starch block $3 \mathrm{~mm}$. $\times 6 \mathrm{~mm} . \times 23 \mathrm{~mm}$. The 3-mm. slice was barely thicker than the thinnest bands observed in the tissues chosen for quantitative analysis (see below). These starch blocks were frozen at $-10^{\circ} \mathrm{C}$. and held for analysis. For analysis, a block was placed in $1.0 \mathrm{ml}$. 0.9 per cent sodium chloride and, after thawing, was homogenized in an all-glass homogenizer to break up the gel structure. The resultant slurry was centrifuged at $4^{\circ} \mathrm{C}$. at $1500 \mathrm{~g}$ for $15 \mathrm{~min}$., and the supernatant material reserved for assay. The substrate mixture for quantitative analysis contained the following final concentrations of reactants (in a volume of $3 \mathrm{ml}$.): $0.033 M$ tris(hydroxymethyl)aminomethane buffer $p \mathrm{H}$ 7.4; $0.003 M$ potassium cyanide; $0.016 M$ substrate material; and $0.00066 M$ DPN or DeamDPN. One-hundred $\lambda$ of the above supernatant material was added to a cuvette containing all reactants except DPN or DeamDPN. After $30 \mathrm{sec}$. the reaction was started by the addition of the appropriate nucleotide. Change in optical density due to the formation of reduced nucleotide was measured at $340 \mathrm{~m} \mu$ over a 1 -min. period at $25^{\circ} \mathrm{C}$., using a Beckman Model DU spectrophotometer. Optical density change was linear over this period of measurement. No-enzyme and no-substrate controls were provided. Quantitative determinations were made in duplicate on material derived from a minimum of 2 electrophoretic separations. All quantitative results are reported as mean values.

Methods of cytochemical study of lactic dehydrogenase distribution in tissue sections were identical to those employed by Allen and Slater (1961), except that $0.025 M$ tris(hydroxymethyl)aminomethane buffer was substituted for phosphate buffer.

\footnotetext{
* Obtained from Sigma Chemical Co., St. Louis, Mo.

† Obtained from the California Corporation for Biochemical Research, Los Angeles, Calif.

$\ddagger$ Obtained from Pabst Brewing Co., Newark, N.J.

$\$$ Obtained from Nutritional Biochemicals Corp., Cleveland, Ohio.
} 


\section{Results}

Tissue specific palterns of multiple forms of lactic dehydrogenase. Examination of the major organs of the mouse indicated a high degree of heterogeneity in terms of the array of multiple forms of lactic dehydrogenase possessed by a particular structure. On the basis of this comparative study it was possible to distinguish 9 electrophoretically distinct sites of lactic dehydrogenase activity. No single tissue or organ possessed all 9 sites, and none failed to show at least 1 site. On the basis of this comparative study it was possible to choose 4 tissues that showed 1 or more of the 9 sites in moderate to high activity. This representative set included skeletal muscle, large intestine, kidney, and testis. The distribution of sites of lactic dehydrogenase activity in these representative tissues is shown in FIGUREs 1 and 2. Sites A2 and A4, derived from large intestine, were close in their electrophoretic mobility to sites A1 (skeletal muscle) and A3 (skeletal muscle). However, study of starch slabs containing half-paper inserts and comparing kidney with large intestine or skeletal muscle with large intestine clearly indicated a difference in the mobilities of sites $\mathrm{A} 1$ and $\mathrm{A} 2$, and $\mathrm{A} 3$ and $\mathrm{A} 4$. Site $\mathrm{C} 1$ was very slow migrating and remained near the origin. The separation of the remaining sites proved no problem. It is probable that the 9 electrophoretically distinct sites separated represent the minimum number since, in certain cases, the presence of diffuse bands precluded positive identification of site position. Thus placenta, mammary gland, coagulating gland, seminal vesicle, parotid gland, and liver possessed diffuse sites in the areas occupied by sites A1 and A2 or A3 and A4 that were impossible to assign to one position or another.

The substrate specificity of the nine sites of lactic dehydrogenase activity. Study of the substrate specificities of the sites of lactic dehydrogenase depended in part upon the ability of the cytochemical reaction system to reflect activity levels accurately. Visual grading of site activity was based upon a scale in which grade 1 represented highest activity and grade 5 represented barely detectable activity. The results of such visual grading when $(l)$ lactic acid was the substrate material may be seen in FIGURE 1 , to which the intensity of the sites shown photographically in FIGURE 2 may be compared. Thus, sites A1, A6, and A7 showed comparable activity of grade 1; site C1 showed slightly lower activity of grade 2; sites A2, A4, A5 showed activity of grade 3; and sites $\mathrm{C} 2$ and $\mathrm{A} 3$ showed activity of grade 4 . Quantitative estimations of eluted enzyme (TABLE 1) closely paralleled estimations of band intensity by visual inspection.

When the activities of the various sites against $(d l)$ alpha-hydroxybutyric acid were compared to the activities against $(l)$ lactic acid, distinct differences in substrate specificity among the sites became apparent (FIGURE 1). Activity of all sites with the exception of $\mathrm{C} 2$ was either depressed or abolished by the substitution of $(d l)$ alpha-hydroxybutyric acid. Site $\mathrm{C} 2$ was as active against this latter material as against $(l)$ lactic acid. Sites A2 and A4 showed no detectable activity against $(d l)$ alpha-hydroxybutyric acid. The remaining sites showed easily discernible but diminished activity against (dl) alphahydroxybutyric acid. Quantitative determinations confirmed the trend of the cytochemical observations (TABLE 1). 


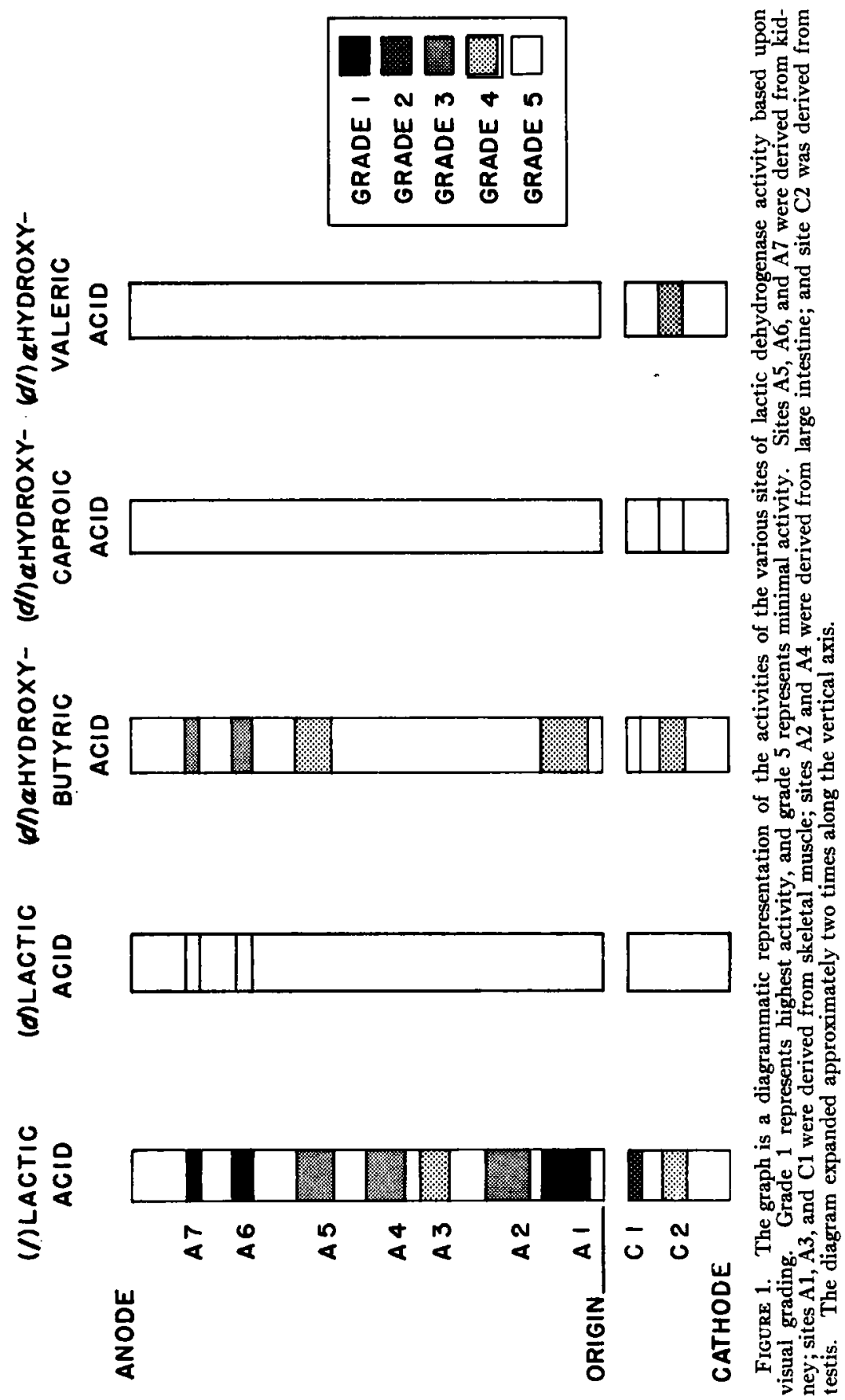




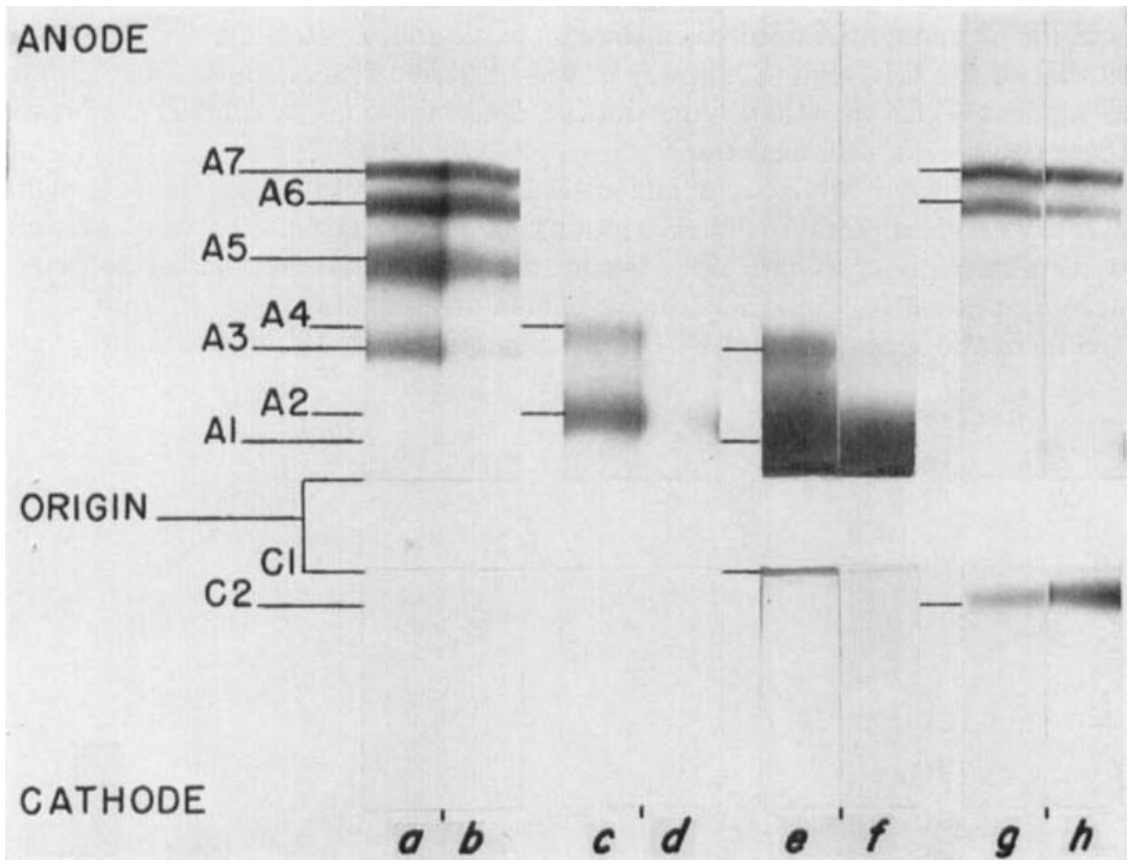

FigURE 2. The activities of the sites of lactic dehydrogenase against $(l)$ lactic acid in the presence of DPN (left member of each pair) and DeamDPN (right member of each pair). Strips $a$ and $b$ derived from kidney; strips $c$ and $d$ derived from large intestine; strips $e$ and $f$ derived from skeletal muscle; and strips $g$ and $h$ derived from testis. Enlarged $1: 1$ from 35 $\mathrm{mm}$. negative material. All conditions of exposure and processing were held to constant values.

TABLE 1

Activity of Eluted Enzyme Against Various Substrates

\begin{tabular}{c|c|c|c|c|c}
\hline \hline Site & $(l)$ Lactic acid & $\begin{array}{c}(d) \text { Lactic acid } \dagger \\
(\%)\end{array}$ & $\begin{array}{c}(d l) \alpha \text { Hydroxy } \\
\text { butyric acid } \\
(\%)\end{array}$ & $\begin{array}{c}(d l) \alpha \text { Hydroxy- } \\
\text { caproic acid } \\
(\%)\end{array}$ & $\begin{array}{c}(d l) \alpha \text { Hydroxy- } \\
\text { valeric acid }{ }^{(\%)} \\
(\%)\end{array}$ \\
\hline A7 & 0.017 & 0 & 20.1 & 0 & 0 \\
A6 & 0.018 & 0 & 14.8 & 0 & 0 \\
A5 & 0.009 & 0 & 9.1 & 0 & 0 \\
A4 & 0.007 & 0 & 0 & 0 & 0 \\
A3 & 0.006 & 0 & 8.1 & 0 & 0 \\
A2 & 0.008 & 0 & 0 & 0 & 0 \\
A1 & 0.040 & 0 & 12.3 & 0 & 0 \\
C1 & 0.012 & 0 & 26.2 & 0 & 0 \\
C2 & 0.007 & 0 & 87.5 & 128.0 & 86.0 \\
\hline
\end{tabular}

* Activity expressed as change in optical density per minute per $100 \lambda$ eluate.

$\dagger$ Activity against this substrate expressed as per cent of activity against $(l)$ lactate control. 
Substitution of $(d l)$ alpha-hydroxycaproic acid or ( $d l)$ alpha-hydroxyvaleric acid for $(l)$ lactic acid resulted in the loss of all anodal sites of activity, as well as that of site $\mathrm{C} 1$. Site $\mathrm{C} 2$, however, was as active against these substitutions as against $(l)$ lactic acid. Quantitative determinations (TABLE 1) confirmed these cytochemical observations.

Substitution of $(d)$ lactic acid for $(l)$ lactic acid resulted in the loss of all activity except at sites A6 and A7 (FIGURE 1). These latter sites showed barely detectable activity (Grade 5). Quantitative determinations failed to detect activity against $(d)$ lactic acid on the part of any site (TABLE 1 ).

Substitution of $(d l)$ alpha-hydroxyisovaleric acid, $(d l)$ alpha-hydroxyiso-

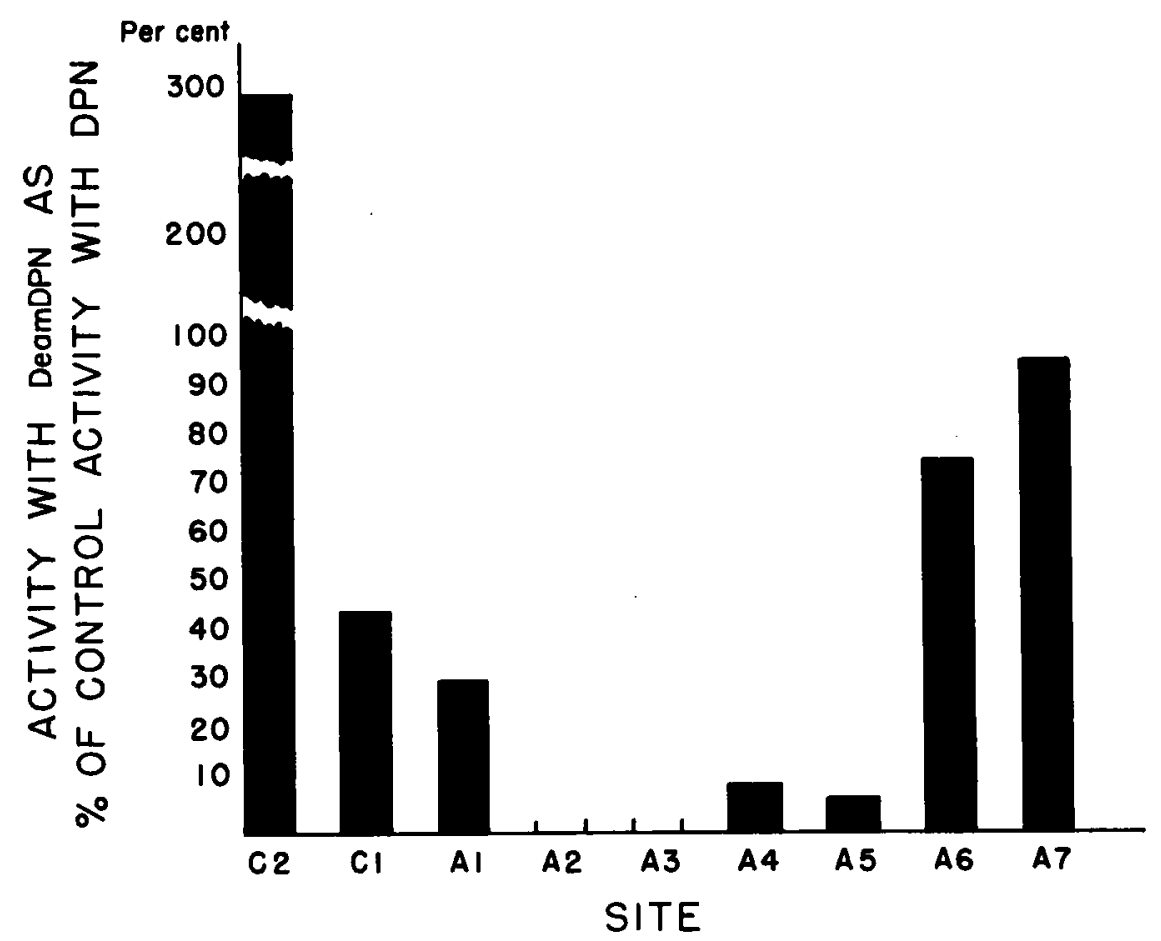

FIGURE 3. The activities of the various sites against (l) lactic acid in the presence of DeamDPN expressed as per cent of activity of the site against $(l)$ lactic acid in the presence of DPN. The derivation of sites is as in FIGURE 1.

butyric acid, or $(d l)$ beta-phenyllactic acid for $(l)$ lactic acid resulted in the loss of all anodal sites, as well as site $\mathrm{C} 1$. Cathodal site $\mathrm{C} 2$ attacked $(d l)$ alpha-hydroxyisovaleric acid at a rate slightly less than that against $(l)$ lactic acid. Activity against ( $d l$ ) beta-phenyllactic acid was of low order (grade 5), and $(d l)$ alpha-hydroxyisobutyric acid yielded no perceptible reaction. Quantitative determinations were not made with this latter group of materials.

Effect of deamino-diphosphopyridine nucleotide upon the activity of the lactic dehydrogenase sites. Cytochemical comparison of the activities of the various sites against $(l)$ lactic acid in the presence of DPN or DeamDPN indicated differences to the extent that the analogue could substitute for the natural 
cofactor. Sites A7, A6, and A1 showed equal cytochemical acitvity (FIGURE 2) with both nucleotides. Sites A5 and C1 showed moderate depression of activity with DeamDPN. Sites A4, A3, and A2 were drastically inhibited by substitution of the analogue. Site $\mathrm{C} 2$, in contrast, was markedly enhanced in activity when DeamDPN was used. Quantitative determinations confirmed and extended these cytochemical observations (FIGURE 3). In this case the activities of sites $\mathrm{A} 7$ and $\mathrm{A} 6$ were seen to be only slightly affected by the substitution of DeamDPN, but the activities of sites A2, A3, A4, and A5 were

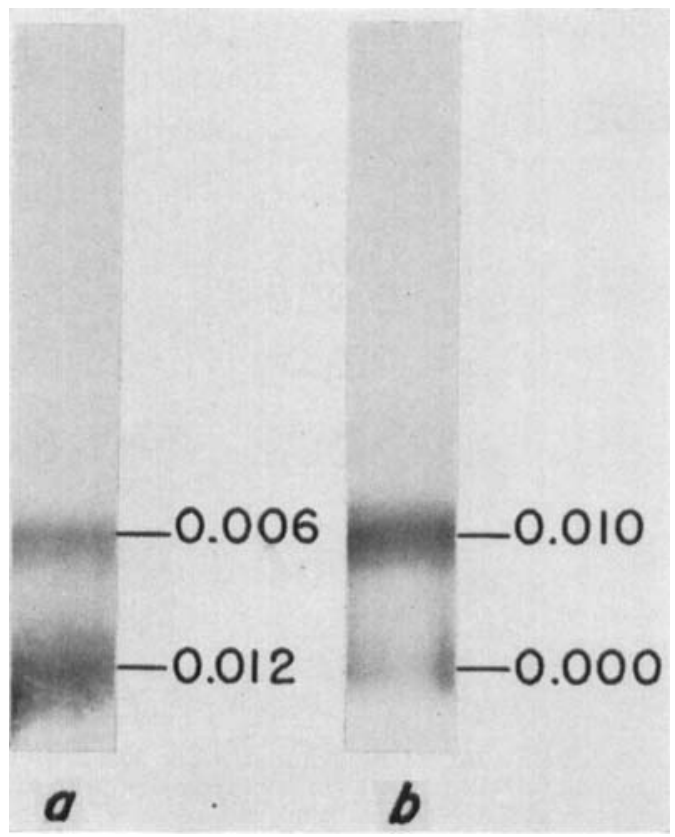

Figure 4. The comparison of activities against $(l)$ lactic acid of sites derived from the mammary gland of an ovariectomized animal $(a)$ with activities of sites derived from the mammary gland of a lactating animal $(b)$. The figures to the right of each column refer to the change in optical density per minute produced by $100 \lambda$ of eluted enzyme in quantitative determinations. Enlarged 1:1 from $35 \mathrm{~mm}$. negative material. The conditions of exposure and development are identical to those employed in FIGURE 2.

drastically depressed. Sites $\mathrm{C} 1$ and A1 showed moderate inhibition of activity with DeamDPN. The activity of site $\mathrm{C} 2$ was increased by nearly threefold when the analogue was substituted for DPN.

The effect of inhibitors upon the activities of the lactic dehydrogenase sites. Inclusion of $0.001 \mathrm{M}$ pyruvic acid or oxamic acid in cytochemical substrates containing DPN and $(l)$ lactic acid resulted in general depression of the activities of the various sites. No differential effect of these materials could be discerned by visual inspection.

The heterogeneity of the response of the various sites to altered physiological conditions. Examination of certain tissues under altered physiological conditions suggested that the response of the various sites was heterogeneous. Comparison of lactating mammary gland with mammary gland from ovari- 
ectomized animals (FIGURE 4) indicated that the sites in this tissue responded differently to these conditions. Both cytochemical and quantitative determinations indicated that the slowest migrating site had highest activity in tissue from ovariectomized animals, whereas the faster migrating site showed highest activity in tissue from lactating animals. Thus there was a complete reversal of the quantitative relations between these sites under the differing physiological conditions. The diffuse nature of the sites in mammary gland precluded definite location as to position. Attempts to visualize shifts in activity within or between cells in sections of mammary gland under these conditions met with failure.

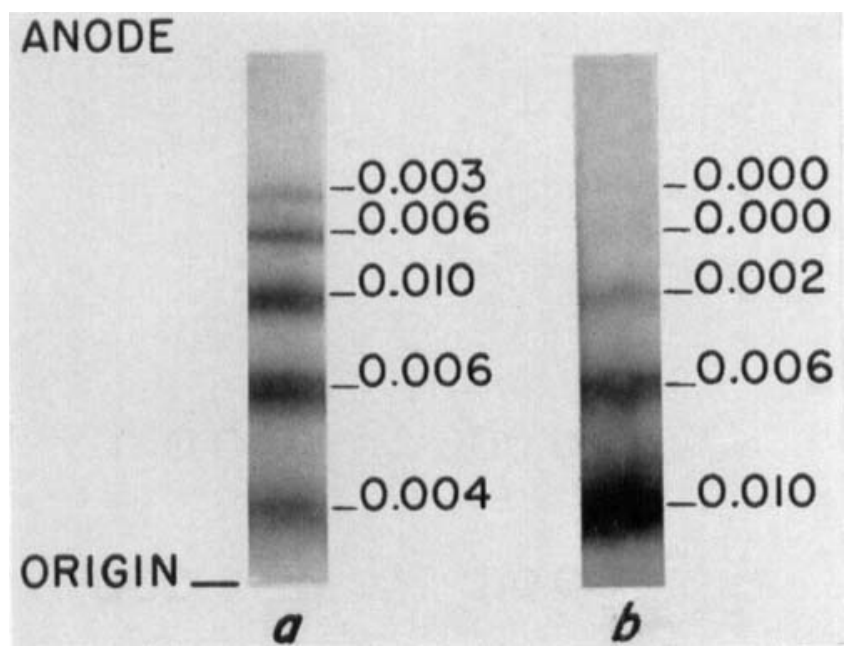

Figure 5. The comparison of activities against $(l)$ lactic acid of sites derived from uter from ovariectomized animals $(a)$ with activities of sites derived from uteri from bvariectomized animals treated with $1 \mu \mathrm{gm}$. estradiol benzoate daily for 5 days $(b)$. The figures to the right of each column refer to the change in optical density per minute produced by $100 \lambda$ of eluted enzyme in quantitative determinations. The sites represented are A1, A3, A5, A6, and A7. Land Polaroid direct positive 1:1, band intensities are not strictly comparable to those in FIGURES 2 and 4.

In another instance, when the uterus from ovariectomized animals was compared to the uterus from ovariectomized animals that had been injected with 1 $\mu \mathrm{gm}$. estradiol benzoate daily for a week, a high degree of heterogeneity in the response of various sites of lactic dehydrogenase activity was noted (FIGURE 5). Site A1 in tissues from estrogen-replaced animals was markedly increased in activity, site $\mathrm{A} 2$ showed activity equal to that seen in ovariectomized animals, and the activities of sites A3, A4, and A5 were greatly depressed when compared to ovariectomized controls. Attempts to follow similar heterogeneity of response on the part of component cells in the uterus under these treatment conditions, using microscopic cytochemical methods, revealed no clear-cut changes in activity that could be related to the changes noted in electrophoretic studies.

In the case of epididymis, comparison of material derived from normal males 
and from males subject to section of the vasa efferentia indicated that this treatment resulted in the total loss of site C2 (FIGURE 6), but had no effect upon the activity of any other site. Identical results were obtained when epididymides from castrated animals were compared to those from normal animals.

The possibility that certain sites may be localized to specific regions of cells. In

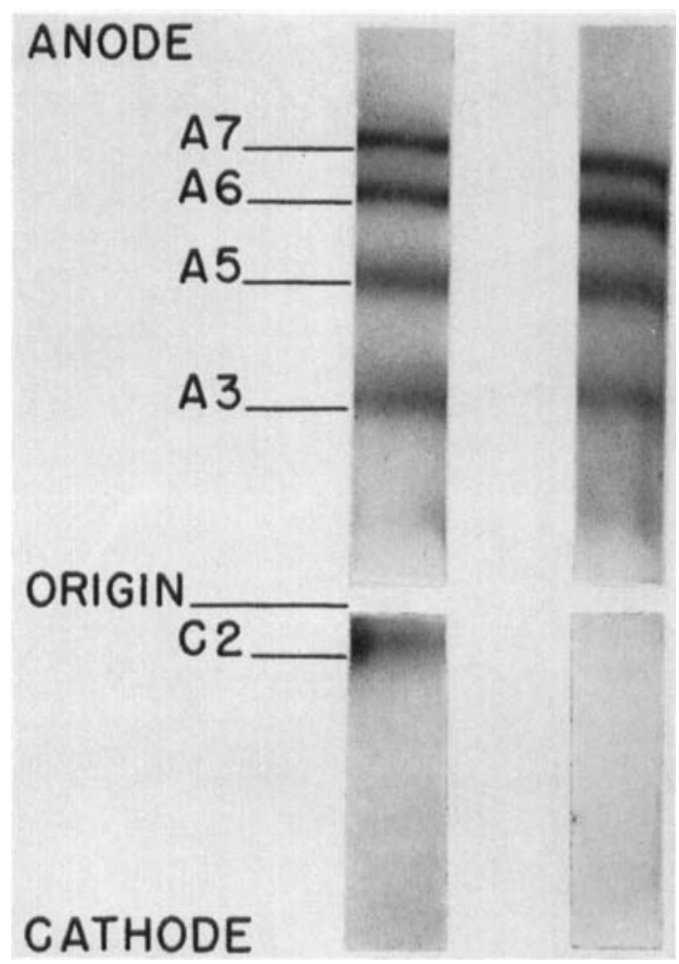

FIGURE 6. The comparison of activities against $(l)$ lactic acid of sites derived from epididymides from normal animals (left) and from animals vasectomized 30 days (right). Enlarged 1:1 from $35 \mathrm{~mm}$. negative material. The photographic representation of site intensities is not comparable to that in FIGUREs 2 and 3 , due to the necessity of processing alterations to show sites with weak activity.

the case of epididymis it appeared that a site visualized in electrophoretic studies could be assigned to a particular cellular locale. Previous work (Allen and Slater, 1961) demonstrated a very distinct localization of lactic dehydrogenase activity to the apical regions of epithelial cells lining the body segment of the epididymal canal (FIGURE 7a). In other portions of the organ, activity was of moderate levels and was distributed homogeneously in the cytoplasm of epithelial cells. Following section of the vasa efferentia (henceforth referred to as vasectomy) for a period of 14 days, the apical activity in the body cells was lost, but levels of activity in the remaining cytoplasm were not affected (FIGURE 7c). Quantitative studies confirmed these cytochemical observations. The loss of epididymal site $\mathrm{C} 2$, previously noted, following vasectomy suggested 
that a relationship might exist between this site and the apically distributed lactic-dehydrogenase activity observed in sections of epididymis.

Support for this contention was derived from studies of the substrate specificity of the apical lactic dehydrogenase in sections of epididymis in comparison with the substrate specificity of epididymal site C2. Substrate properties identical to those observed for testis site $\mathrm{C} 2$ were noted for epididymal site $\mathrm{C} 2$,
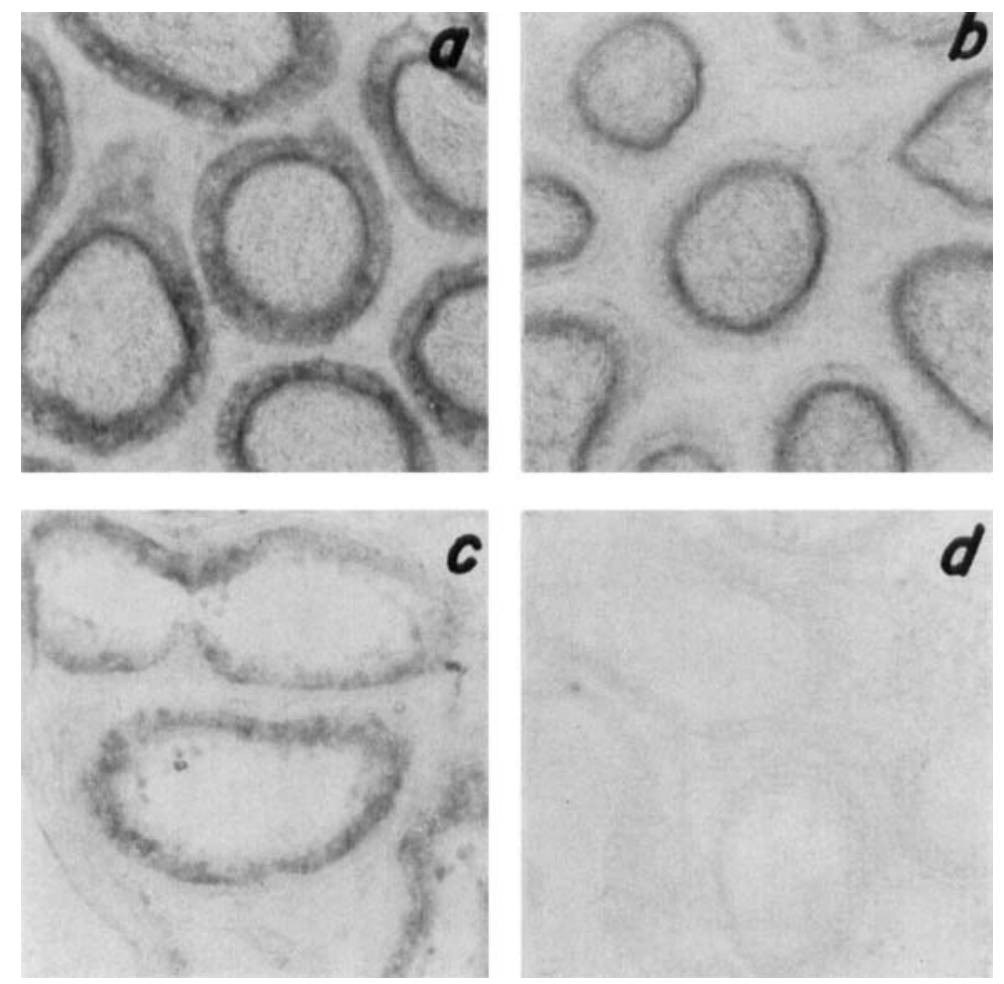

FIGURE 7. (a) The distribution of activity against $(l)$ lactic acid in the epithelial cells of the body segment of epididymis from a normal animal. A marked apical distribution of activity is noted in these cells. The more basal cytoplasm contains moderate levels of homogeneously distributed activity. Nuclei, connective tissue cells, and sperm cells are unreactive. Five min. incubation at $37^{\circ} \mathrm{C}$. with nitro-blue tetrazolium, $10 \mu$ cryostat section. Land Polaroid direct positive, Wratten G filter, $\times 165$.

(b) The distribution of activity against (dl) alpha-hydroxycaproic acid in the epithelial cells of the body segment of epididymis from a normal animal. A very distinct apical distribution of activity is seen. The more basal portions of the cytoplasm are unreactive in contrast to the moderate activity seen in the same region in $a$. Nuclei, connective tissue cells, and sperm cells are unreactive. The preparation and photography are identical to that used in a.

(c) The distribution of activity against $(l)$ lactic acid in the body cells of epididymis from an animal vasectomized for 30 days. The apical distribution of enzyme is no longer visible (compare to a) but moderate levels of activity are homogeneously distributed in the general cytoplasm. Nuclei and connective tissue cells are unreactive. The preparation and photography are identical to that used in $a$.

(d) The distribution of activity against (dl) alpha-hydroxycaproic acid in the body cells of epididymis from an animal vasectomized for 30 days. There is a complete loss of reaction in all tissue components. The preparation and photography are identical to that used in $a$. 
except that activity levels were lower. The anodal sites of epididymis were inactive against any of the test substrates [except for $(l)$ lactic acid] when incubation was for 30 min. Sites A6 and A7 showed barely perceptible activity with $(d l)$ alpha-hydroxybutyric acid after 1 hour incubation. Low levels of activity in epididymal sites precluded elution and quantitative measurement of site activities. When similar tests of substrate specificity were made using sections of epididymis from normal animals, the apical regions of the epithelial cells lining the body segment of the canal showed equal intensity of reaction with $(l)$ lactate, $(d l)$ alpha-hydroxybutyric acid, $(d l)$ alpha-hydroxyvaleric acid, and $(d l)$ alpha-hydroxycaproic acid (FIGURE $7 b$ ). This cellular region did not react with $(d)$ lactic acid. In all of these cases, with the exception of $(l)$ lactic acid, no other area in these cells was reactive (FIGURE $7 b$ ). Other cells, not restricted to the body region of the epididymis, and which gave moderate homogeneously distributed reactions with $(l)$ lactic acid, were completely unreactive with the other test substrates.

Tissues from vasectomized animals were completely unreactive (FIGURE $7 d$ ) with the test substrates, with the exception of $(l)$ lactic acid. This latter material yielded a moderate reaction in the general cytoplasm, which was comparable to that seen in these areas in tissues from normal control animals (FIGURE 7c).

Experiments were carried out to determine if the loss of site $\mathrm{C} 2$ in vasectomized animals might be due to loss of sperm cells. When equal aliquots of whole homogenates, supernatant fractions (derived as usual), and washed sediments derived from the original centrifugation (washed 3 times in 0.9 per cent sodium chloride, centrifuged at $1000 \times \mathrm{g}$ for $10 \mathrm{~min}$. after each wash, and resuspended in a volume of $\mathbf{0 . 9}$ per cent sodium chloride equal to the original aliquot) were compared it was found that no activity was associated with the washed sediment. Thus it appears unlikely that the loss of site C2 in electrophoretic studies of epididymides from vasectomized animals is due simply to absence of sperm cells.

\section{Discussion}

The data accumulated in this study suggest that a considerable degree of heterogeneity exists between the various molecular forms of lactic dehydrogenase in the tissues of the mouse. In terms of substrate specificity it appears that these molecular forms do not have identical activities against the test substrates employed. The most conservative interpretation, in which minor differences in activity are ignored, indicates that the sites of lactic dehydrogenase discriminated may be separated into 3 groups or sets. Site C2 is clearly to be differentiated from the remaining sites on the basis of its capacity to dehydrogenate all of the test substrates, with the exception of $(d)$ lactic acid. Similarly sites $\mathrm{C} 2$ and $\mathrm{C} 4$ may be differentiated from their congeners on the basis of their failure to oxidize any test substrate with the exception of $(l)$ lactic acid. The remaining sites form a group of enzymes that show appreciable activity only against $(l)$ lactic acid and $(d l)$ alpha-hydroxybutyric acid. Quantitative determinations indicated, within the group, variation in the relative rates with which $(d l)$ alpha-hydroxybutyric acid was dehydrogenated. The validity of these minor variations must await further determinations and 
statistical analysis. A suggestion of cleavage within this latter group rests in the capacity of sites A6 and A7 to oxidize (d) lactic acid. All other sites failed to react with this compound. These results are in contradistinction to those of Markert and Mpller (1959). These investigators felt that activity of the multiple forms of lactic dehydrogenase was identical against members of a similar battery of test substrates. The differences obtained in the present study may be due to the use of an improved substrate mixture for visualization of activity on starch. The employment of phenazine methosulfate and nitroblue tetrazolium in the Dewey and Conklin (1961) substrate would be expected to yield more critical rendering of activity values, due to the use of a more sensitive electron acceptor (NBT) and an artificial electron carrier (PMS) rather than natural diaphorase. Quantitative studies confirmed the accuracy with which the cytochemical system reflected activity levels of the various sites. At the present time no other comparable studies are known to me that deal with the substrate specificity of multiple forms of lactic dehydrogenase derived from vertebrate tissues. However, Singer et al. (1960) suggest that there may be multiple forms of lactic dehydrogenase in yeast, which are distinguishable on the basis of their capacity to oxidize $(l)$ lactic acid and (d) lactic acid when different electron acceptors are used. In the case of a hexosephosphatase derived from E. coli, Rogers and Reithel (1960) have been able to identify 3 molecular forms of the enzyme, each with different relative activities against various substrates. In other cases, multiple forms of enzymes separated by methods similar to those used in the present study appear to have identical substrate specificities. Press et al. (1960) found cathepsin D to occur in 10 electrophoretically distinct forms with identical substrate capacities. The multiple forms of esterases studied in Tetrahymena by S. L. Allen (1961) also appear to have identical substrate properties within an isozymic group. Certainly the problem of substrate specificity of multiple forms of enzymes is far from resolved. It is likely that a considerable degree of variation in the extent of substrate specificity between multiple forms of different enzymes exists, and no simple statement of these relations will suffice for their description.

The use of DeamDPN served to further differentiate the multiple forms of lactic dehydrogenase, and indicated that cleavage within the sets mentioned above is possible. The activities of sites A6 and A7 were only slightly affected by the substitution of the analogue, whereas activity in sites A2, A3, A4, and A5 was drastically reduced. Sites $\mathrm{A} 1$ and $\mathrm{C} 1$ were moderately inhibited in activity by the substitution, but the activity of site $\mathrm{C} 2$ was elevated. These results further contrast the properties of site $\mathrm{C} 2$, and also point to a difference in the properties of sites $\mathbf{A} 6$ and $\mathbf{A 7}$ and the properties of the remaining sites. The difference in properties of sites A6 and A7 was suggested by their capacity to oxidize (d) lactic acid (see above). The use of cofactor analogues in the study of dehydrogenases separated electrophoretically has not been reported previously. However Kaplan et al. (1960) used analogues of DPN to successfully distinguish lactic dehydrogenases derived from the same tissues of different species and different tissues of the same species. These results are interpretable on the basis that multiple forms of lactic dehydrogenases exist that have 
different capacities for interaction with various analogues of nucleotide cofactors.

In the present study the use of inhibitors of lactic dehydrogenase failed to distinguish between the various multiple forms of lactic dehydrogenase. In other cases, inhibitor studies have served to differentiate between lactic dehydrogenases of different tissues (Pfleidlander $e t$ al., 1958).

An important distinction between multiple forms of an enzyme may reside in differential responses to changes in physiological environment. The reciprocal behavior of the sites of lactic dehydrogenase activity seen when comparing mammary tissue from lactating and ovariectomized animals is illustrative of such a situation. Also the heterogeneity of site response demonstrated in comparisons of uterus from ovariectomized and estrogen-treated ovariectomized animals indicates a differential response of the components of a multiple enzyme system to physiological change. It is probable that these differential responses have important significance in terms of cellular function, and they may reflect the action of microenvironmental infiuences upon enzymes situated in different regions of the cell or, perhaps, in different cells. Similar heterogeneity of response between members of the family of nonspecific esterases resident in epididymis has been demonstrated by Allen and Hunter (1960). The changes in lactic dehydrogenase constituents of tissues during differentiation reported by Markert and Møller (1959) are doubtless due to analogous environmental alterations. Such differential responses suggest that multiple forms of enzymes, although they may have similar substrate specificities, may be instrumental in different physiological functions.

A final point related to the heterogeneity of multiple forms of lactic dehydrogenase (and other enzymes as well) is the possibility that various constituents of these groups are situated at different sites within the cell. Such topological heterogeneity might be expected to have important implications in terms of the activity of an enzyme within the cell. A differential situation could result in the exposure of an enzyme to different microenvironmental influences that would be expected to affect its function. Also specific localization of an enzyme could result in channeling biochemical reaction sequences. Indeed a great deal of present-day cytological research demonstrates the restriction of enzymatic capacities to this or that cell structure. The research of Greene (1961) demonstrates most clearly the specific situation of macromolecules and the importance of this for the completion of a biochemical reaction sequence (electron transport and oxidative phosphorylation). The cytochemical studies reported in the present paper indicate that one of the sites of activity seen in electrophoretic preparations (C2) may be restricted to a specific cellular region. Restriction of a particular member of an isozymic set to a specific cellular region has also been reported for esterase in Tetrahymena (S. L. Allen, 1961). Nace et al. (1961) have been able to localize serologically distinct lactic dehydrogenases to different regions of the frog oocyte. It is possible that many of the multiple forms of enzymes separable by electrophoresis may represent enzymes with specific cellular localizations.

The heterogeneity of distribution of multiple forms of lactic dehydrogenase seen between various tissues and organs raises the question that each site may 
be resident in a given cell type and that the observed heterogeneity within tissues is due simply to a multiplicity of component cells. It is unlikely that this is true, as studies carried out on cultured fibroblasts (Tsao, 1960) showed a multiplicity of electrophoretically distinct lactic dehydrogenases. Also clonal lines of Tetrahymena (S. L. Allen, 1961) contain a number of esterase isozymes, and Nace et al. (1960) found four serologically distinct lactic dehydrogenases within frog ova.

On the basis of these studies it appears that a considerable degree of heterogeneity exists between different members of a multiple molecular series of lactic dehydrogenases. The differences between members of such a series are not as profound as those existing between hydrolases with low degree of substrate specificity (Markert and Hunter, 1959; Hunter and Burstone, 1960). In agreement with Markert and Møller (1959) it is felt that a continuum of properties does not exist between one enzyme and the next. However, it is felt that pronounced differences in properties do exist between members of the multiple series of lactic dehydrogenases from mouse tissues. These differences are extensive enough in certain instances (site $\mathrm{C} 2$ versus sites $\mathrm{A} 2$, and $\mathrm{A} 4$, and the remaining sites) to suggest that, within the lactic dehydrogenase series, sets or groups of enzymes exist with similar properties. It is perhaps proper to term these groups isozymic sets to indicate that the members possess similar characters. It is evident that the characterization of electrophoretically distinct macromolecules must rest upon several points of comparison rather than upon a single criterion such as substrate specificity.

\section{Summary}

The multiple forms of lactic dehydrogenase in tissues of the mouse have been investigated, using starch-gel electrophoresis combined with cytochemical and quantitative methods of analysis. A survey of the major organs of the mouse indicated that at least nine electrophoretically distinct lactic dehydrogenases were demonstrable. The properties of these sites were studied, using extracts of skeletal muscle, kidney, testis, and large intestine. The substrate specificities of the various sites, demonstrated in electrophoretic studies, differed in their capacity to oxidize $(l)$ lactic acid, $(d)$ lactic acid, $(d l)$, alpha-hydroxybutyric acid, $(d l)$ alpha-hydroxycaproic acid, and $(d l)$ alpha-hydroxyvaleric acid. Thus one site had equal activity against all substrates with the exception of $(d)$ lactic acid. Two sites oxidized only $(l)$ lactic acid. The remaining sites had variable activity against $(d l)$ alpha-hydroxybutyric acid, two of these oxidized $(d)$ lactic acid, and none was active against $(d l)$ alpha-hydroxycaproic acid, or ( $d l)$ alphahydroxyvaleric acid. The use of deamino-diphosphopyridine nucleotide served to contrast further these multiple forms of lactic dehydrogenase. Certain sites were affected only slightly by the substitution of the analogue, others were drastically depressed in activity, and one site was increased in activity.

The behavior of the various sites to physiological modification was examined. The behavior of the various sites to such modification was heterogeneous. In one case, by taking advantage of physiological changes and substrate specificity differences, it was possible to localize a site observed in electrophoretic prepara- 
tions to a particular locale within the epithelial cells lining the body segment of the epididymal canal.

These results are discussed in terms of the physiological significance of multiple forms of enzymes for tissue and cell function.

\section{References}

AlleN, J. \& R. Hunter. 1959. A histochemical study of enzymes in the epididymis of normal, castrated and hormone replaced castrated mice separated by zone electrophoresis in starch gels. J. Histochem. Cytochem. 8: 50-57.

Allen, J. \& J. Slater. 1961. A cytochemical analysis of the lactic dehydrogenase-diphosphopyridine nucleotide-diaphorase system in the epididymis of the mouse. J. Histochem. Cytochem. 9: 221-233.

ALLEN, S. L. 1961. Genetic control of the esterases in the protozoan Tetrahymena pyriformis. Ann. N.Y. Acad. Sci. 94(3): 753-773.

DEWEY, M. \& J. ConkLIN. 1961. Starch gel electrophoresis of lactic dehydrogenases from rat kidney. Proc. Soc. Exptl. Biol. Med. 105: 492-494.

GreEN, D. \& Y. HATEFI. 1961. The mitochondrion and biochemical machines. Science. 133: $13-19$.

HUnter, R. \& M. Burstone. 1960. The zymogram as a tool for the characterization of enzyme substrate specificity. J. Histochem. Cytochem. 8: 58-62.

Kaplan, N., M. Ciotti, M. Hamolshy \& R. Bieber. 1960 . Molecular heterogeneity and evolution of enzymes. Science. 131: 392-397.

MaRKERT, C. \& F. M $\phi$ LL.er. 1959. Multiple forms of enzymes: tissue, ontogenetic, and species specific patterns. Proc. Natl. Acad. Sci. 45: 753-763.

MarkerT, C. \& R. Hunter. 1959. The distribution of esterases in mouse tissues. J. Histochem. Cytochem. 7: 42-49.

Nace, G., T. Suyama \& N. Smith. Early development of special proteins. Symposium of Internat. Inst. of Embryol. Pallanza, Italy. In press.

Pflemerer, G., D. Jeckel \& T. Wieland. 1958. Uber den Wirkungsmechanismus der Milchsaure-dehydrogenasen. II. Zur Frage des Zinkgehalts und der SH-Gruppen. Biochem. Z. 330: 296-302.

Press, E., R. Porter \& J. Cerbra. 1960. The isolation and properties of a proteolytic enzyme, cathepsin $\mathrm{D}$, from bovine spleen. Biochem. J. 74: 501-514.

Rogers, D. \& F. ReIthel. 1960. Acid phosphatases of Escherichia coli. Arch. Biochem. Biophys. 89: 97-104.

Singer, T. P., E. Kearney, D. Gregolin, E. Boeri, M. Rippa \& E. Ford. 1960. On the multiplicity of lactic dehydrogenase in yeast. Biochem. Biophys. Research Com. 3: $428-434$.

SmithIES, O. 1959. Zone electrophoresis in starch gels and its application to studies of serum proteins. In Advances in Protein Chemistry. 14: 65-113. C. Anfinsen, $M$. Anson, K. Bailey, and J. Edsall, Eds. Academic Press. New York, N.Y.

Tsao, M. 1960. Heterogeneity of tissue dehydrogenases. Arch. Biochem. Biophys. 80: 234-238.

\section{Discussion of the Paper}

A. Samuels (Department of Pathology, Dartmouth Medical School, Hanover, N.H.) : Was it possible to make any estimate of the time course of disappearance of any of the fractions during physiological manipulation, so that a comparison of this time and the turnover time of the protein could be made? This could possibly lead to insight as to whether the synthesis or the conformation of the protein was affected by the treatment. 